\title{
Retroinformática para la enseñanza y el aprendizaje en la Ingeniería Informática
}

\author{
Xavier Molero Ana Pont, Antonio Robles y Milagros Martínez \\ Escola Tècnica Superior d'Enginyeria Informàtica \\ Departament d'Informàtica de Sistemes i Computadors \\ Universitat Politècnica de València \\ \{xmolero, apont, arobles, mimar\}@disca.upv.es
}

Resumen En este trabajo mostramos cómo a través de la retroinformática tratamos de motivar a nuestros estudiantes en el estudio de la asignatura Estructura de Computadores. A ello contribuye el Museo de Informática de la Universitat Politècnica de València que se ha convertido durante los dos últimos cursos en un instrumento didáctico adicional para la docencia, además de cumplir con la importante misión de difundir la cultura e historia de la informática entre los estudiantes de grado de ingeniería informática. La experiencia se ha llevado a cabo durante el pasado y el actual curso con los alumnos de segundo año del mencionado grado y en el ámbito de las tareas académicas de la asignatura. El artículo explica los detalles de la articulación de las actividades en una asignatura con más de cuatrocientos alumnos matriculados y organizados en siete grupos de aula, lo que ya es de por sí un reto nada desdeñable. Parte de estas actividades se han llevado a cabo directamente por parte del profesorado y otras lo han sido de forma autodirigida mediante cuestionarios en papel y herramientas online. Por último, este trabajo muestra la manera en que se han evaluado las experiencias en ambos cursos y las conclusiones extraídas de las mismas para poder mejorarlas en futuras ediciones e incorporar nuevos retos para el futuro.

Palabras clave: motivación, retroinformática, materias básicas, museo de informática, informática y sociedad, historia de la informática.

\begin{abstract}
In this work we show how retrocomputing can be used to motivate and encourage our students to address Computer Organization subject. The Museum of Informatics of the UPV contributes to this purpose and during the last two academic years has become an additional teaching tool for the mentioned subject besides of its important mission for patrimonial and cultural dissemination among students of Computer Engineering Degree. The experience has been carried out during the last and the current academic year in this degree in the scope of the academic tasks related to the subject. This paper explains how the experience was organized and carried out for more than 400 students organized into 7 class groups, which is by itself an important challenge. Some of the related activities were directly performed by the teaching staff and others
\end{abstract}


were planned in a self-guided way, both using online tools and paper and pencil surveys. Finally, this work show how the experience has been evaluated in both years, the main conclusions got to improve it for new future editions and how to include new challenges.

Keywords: Motivation, retrocomputing, core subjects, Museum of Informatics, Informatics and Society, Computer History

\section{Introducción y motivación}

En la enseñanza de materias básicas de las titulaciones de informática, y en especial de aquellas que tienen como objetivo ofrecer una visión sobre los fundamentos de los computadores y los elementos básicos del hardware y software que los componen, es especialmente difícil dar una visión aplicada y cercana a la realidad de sus contenidos. Conocer los principios básicos del funcionamiento de un computador requiere de una serie de simplificaciones y abstracciones que resultan muy lejanas de la realidad tecnológica actual, pero que son necesarias para crear la base de conocimiento que permita, con posterioridad, abordar conceptos y paradigmas actuales, mucho más complejos y sofisticados.

Esta visión elemental de la máquina resulta muchas veces poco atractiva y desmotivadora para nuestros actuales estudiantes, acostumbrados a utilizar, en su día a día, dispositivos y aplicaciones basados en las últimas tecnologías difícilmente relacionables con los contenidos de la materia. Motivar a los estudiantes para que encuentren atractivas estas materias básicas deviene, por tanto, en una tarea difícil.

Por otra parte, la reducción de horas lectivas llevada a cabo en los recientes planes de estudio, así como la desaparición de las asignaturas de libre elección, hace cada vez más difícil dedicar tiempo a aspectos relacionados con la historia de la informática. Si bien estos aspectos no forman parte directamente de las competencias y habilidades específicas que se esperan conseguir de los estudios, sí son, en nuestra opinión, conocimientos básicos a reivindicar para la formación de los ingenieros informáticos.

Conocer la historia y evolución de cualquier ciencia o técnica no solo ayuda a comprender y contextualizar mejor el presente; su estudio proporciona, a pesar de la dificultad para medir objetivamente este beneficio, un bagaje cultural realmente valioso para nuestros egresados toda vez que permite mejorar la sensibilidad y compromiso con la sociedad y el medio ambiente, unos valores de carácter transversal que el diseño de los actuales planes de estudio suelen precisar y tener en cuenta.

Por todo ello, consideramos que conocer la historia de la informática no es únicamente una forma de motivar a nuestros estudiantes sino que, además, contribuye de forma clara a alcanzar las competencias de la titulación [2009]. En este sentido, el Museo de Informática de la UPV nos brinda una oportunidad única para conseguir un doble objetivo en nuestros alumnos: 
1. Motivar el estudio y facilitar la comprensión de la materia Estructura y Arquitectura de Computadores.

2. Ampliar su cultura informática a través del conocimiento de su historia y evolución tecnológica.

Por ello, al inicio del curso 2013/14 se planificó la visita al Museo de Informática de la UPV como una actividad asociada a la asignatura Estructura de Computadores. Esta actividad se planteó como una tarea más a realizar dentro de la asignatura y con la misma filosofía que el resto de actividades pedagógicas, esto es, con carácter voluntario pero puntuable. A pesar de los problemas propios asociados a la primera vez que se organiza cualquier actividad, la edición del pasado curso nos permitió ver las posibilidades de la retroinformática ${ }^{1}$ como elemento de innovación docente, por lo que en el curso actual 2014/15 la experiencia se ha llevado también a cabo incluyendo nuevas actividades.

En el resto del artículo se indica el contexto de la asignatura, se describe brevemente el Museo de Informática como marco y elemento básico de la actividad, se explica cómo ésta se ha organizado y llevado a cabo en esto dos cursos así como los contenidos de la misma y su conexión con la materia estudiada. Finalmente, se describe cómo se ha valorado el impacto de la experiencia en ambas ediciones, el alcance de los objetivos propuestos y el nivel de satisfacción mostrado por los estudiantes con la misma.

\section{Estructura de Computadores: la asignatura}

La asignatura Estructura de Computadores (EC) del plan de estudios de Grado en Ingeniería Informática que se imparte en la UPV forma parte de la materia de carácter obligatorio denominada Estructura y Arquitectura de Computadores. Esta materia se descompone en dos asignaturas, la mencionada EC, con un peso de 9 ECTS e impartida de manera anual en segundo curso, y la asignatura Arquitectura e Ingeniería de Computadores, de 6 ECTS y ubicada en el primer semestre del tercer curso. La carga anual de la asignatura EC se organiza en 6 ECTS de teoría de aula y problemas y 3 ECTS de laboratorio.

Como base informática previa, los estudiantes han cursado 24 ECTS con carácter de formación básica a través de las asignaturas Fundamentos de Compu-

\footnotetext{
${ }^{1}$ A lo largo de este artículo el prefijo retro se utiliza en castellano como abreviatura de retrospectiva (del latín retrospicere), adjetivo que, según la Real Academia Española, indica que «se considera en su desarrollo anterior», y no como el prefijo latino retroque significa «hacia atrás». La abreviatura retro, en el primer sentido mencionado, es una manera cada vez más utilizada de referir coloquialmente aspectos de un tiempo pasado o que lo evocan. La Wikipedia en inglés (http://en.wikipedia.org) se hace eco ampliamente del término retrocomputing, que define como the use of older computer hardware and software in modern times. Este último es el sentido que le hemos querido dar en este trabajo, en el que nos hemos tomado la licencia de usar el término retroinformática, construida léxicamente igual que la palabra rétro-informatique usada en francés.
} 
tadores, Introducción a la Informática y a la Programación, Tecnología de Computadores y, finalmente, Programación, todas ellas de 6 ECTS.

La competencia indispensable que se pretende alcanzar con la asignatura Estructura de Computadores se cita textualmente en la guía docente [2012] de la misma como: capacidad de conocer, comprender y evaluar la estructura y arquitectura de los computadores, así como los componentes básicos que los conforman.

Para satisfacer la mencionada competencia, esta asignatura comprende el estudio de las distintas unidades funcionales que integran un computador y que hacen posible la ejecución de los programas, prestando especial atención a la íntima relación entre el hardware y el software, así como a la manera en que diferentes estructuras organizativas de las unidades funcionales pueden influir sobre las prestaciones del computador. En este sentido, la asignatura aborda el estudio del procesador, la unidad aritmético-lógica, el sistema de memoria, la unidad de entrada/salida y los dispositivos periféricos. Todo ello se organiza en las siguientes unidades temáticas:

1. El procesador

- Tema 1: La ruta de datos

- Tema 2: Segmentación básica

2. Unidad aritmético-lógica

- Tema 3: Unidad aritmética de enteros

- Tema 4: Unidad aritmética de coma flotante

3. Unidad de memoria

- Tema 5: El sistema de memoria

- Tema 6: Jerarquía de memoria

4. Unidad de entrada/salida

- Tema 7: Adaptadores e interfaces de entrada/salida

- Tema 8: Sincronización de la entrada/salida

- Tema 9: Técnicas de transferencia de la entrada/salida

5. Los periféricos y las estructuras de interconexión

- Tema 10: Dispositivos periféricos

- Tema 11: Estructuras de interconexión

Toda la materia de Estructura y Arquitectura de Computadores impartida en el grado toma el procesador MIPS R2000 como hilo conductor. Ya en la asignatura Fundamentos de Computadores de primer curso se inicia el estudio de este procesador presentando su estructura básica y los rudimentos de su lenguaje ensamblador. De manera similar a la propuesta del libro Estructura y diseño de computadores. La interfaz hardware/software de D.A. Patterson y J.L. Hennessy [2011], el procesador MIPS R2000 y su lenguaje ensamblador forman el sustrato sobre el que se explican las unidades funcionales del computador, su interconexión y los parámetros clave de sus prestaciones, tanto en el aula como en el laboratorio. Para una mayor información sobre la asignatura, metodología docente y evaluación, puede consultarse su correspondiente guía docente [2012].

A pesar de esta visión básica y clásica de la materia que nos ocupa, la asignatura recurre a ejemplos de la tecnología actual tanto para ilustrar los temas de 
memoria, dispositivos periféricos y elementos de interconexión, como para ofrecer una visión evolutiva de los aspectos clave en el diseño de los procesadores.

En este sentido, estamos plenamente convencidos de que la exposición de la retroinformática que ofrece el Museo de Informática de la UPV puede jugar un papel clave para contextualizar los contenidos de la asignatura porque permite situar en el tiempo los avances de la tecnología, tanto en lo referente al hardware como a la programación y al desarrollo de las aplicaciones [2014]. Así, la visión retrospectiva - y no lo olvidemos, también social - que proporciona el museo acerca de la informática puede aprovecharse como un factor más de motivación para el estudio de los computadores.

\section{$3 \quad$ El Museo de Informática}

El Museo de Informática ${ }^{2}$ de la Universitat Politècnica de València ofrece su colección al visitante como un viaje en el tiempo a través de la historia de la informática [2014]. Inaugurado en el año 2001 y, por tanto, uno de los pioneros en España, ha sido reconocido como museo oficial de su comunidad autónoma por su compromiso con la sociedad y su determinada vocación de servicio público. Por otro lado, el museo ha sido admitido recientemente como miembro de la organización ICOM (International Council of Museums ${ }^{3}$ ) de la UNESCO con el fin de participar activamente en la conservación y protección del patrimonio cultural ligado a la informática.

El proyecto de difusión patrimonial del museo se dirige principalmente a los jóvenes estudiantes de nuestro sistema educativo y también al público en general. $\mathrm{Su}$ objetivo primordial es dar a conocer la historia de la informática (orígenes, evolución tecnológica) y, dado que casi todos nosotros somos usuarios asiduos de dispositivos o aplicaciones de carácter informático (internet, redes sociales, ...), el museo pretende incitar a la reflexión crítica sobre otros aspectos menos conocidos, pero de gran importancia para nuestra sociedad, que no dejan de tener relación con la informática (basura electrónica y reciclaje, privacidad de la información, derechos humanos, ...). En el seno del museo se organiza un amplio abanico de actividades pedagógicas y culturales, entre las que destacan las visitas guiadas, los talleres de trabajo (y de juegos) con ordenadores antiguos, los ciclos de cine y los cursos de retroprogramación (por ejemplo, programación en lenguaje BASIC en microcomputadores de 8 bits).

La exposición permanente del museo se organiza en un conjunto de vitrinas y paneles informativos, así como dispositivos de gran tamaño, dispuestos a lo largo de las tres plantas que conforman uno de los edificios en los que se ubican las instalaciones de la Escola Tècnica Superior d'Enginyeria Informàtica. En particular, esta exposición contiene una muestra representativa de la informática de las tres últimas décadas del pasado siglo XX. El criterio expositivo utilizado es el cronológico, aunque sometido a ligeras modificaciones a causa de las restricciones impuestas por el ámbito expositivo disponible. Por otro lado, y ante

\footnotetext{
${ }^{2}$ http://museo.inf .upv.es

3 http://icom.museum
} 
la necesidad de materialidad que una exposición inevitablemente establece, los objetos (tanto ordenadores como otros dispositivos) se acompañan, siempre que ha sido posible, por los programas que usaban, materializados bien mediante las unidades de cinta o disco donde están grabados, bien mediante publicidad o listados de código que sirven de ejemplo. El contexto de cada objeto ha sido rnriquecido, además, con fotografías de personajes, manuales u otros tipos de documentos que ayudan a situar su ámbito de aplicación.

De la informática de finales de los años 70, dominada por las grandes instalaciones corporativas (mainframes), en la exposición se puede contemplar, entre otras, una perforadora de tarjetas, memorias de ferrita, una impresora de líneas y varias unidades de cinta y disco magnéticos de grandes dimensiones. El grueso de la exposición está determinado por la informática doméstica que se gestó en los años 80, momento histórico en el que convivieron un gran número de microcomputadores y aplicaciones de 8 bits. Se pueden ver ejemplares fabricados por empresas como Sinclair, Amstrad, Commodore o Atari; no podían faltar, en esta lista, los ordenadores MSX, el primer IBM PC o el primer Apple Macintosh. De esta época el museo también cuenta con varios minicomputadores, como el DEC PDP-11 y el Nixdorf Quattro/30, que marcan el advenimiento de sistemas informáticos de tamaño medio y precios asequibles capaces de ejecutar aplicaciones antes reservadas de manera exclusiva a los grandes computadores.

Los años 90 están representados por los dos tipos de computadores personales que se adueñaron finalmente de la casi totalidad del mercado: los compatibles con el IBM PC y los computadores fabricados por Apple, utilizados ambos a través de sistemas gráficos basados en ventanas (GUI, Graphical User Interface). Por otro lado, también tienen un papel destacado en el museo las estaciones de trabajo (workstations) aparecidas a finales de la década de los 80 y destinadas principalmente a aplicaciones científicas y técnicas con una gran componente gráfica.

Así mismo, también es posible contemplar la evolución del tamaño de los computadores personales a través de varios ejemplares de portátiles (o transportables, como fueron conocidos en un primer momento). El abanico de artefactos abarca, por otra parte, un conjunto de expositores con un amplio surtido de microprocesadores, módulos de memoria semiconductora, dispositivos de almacenamiento (tarjetas perforadas, discos, cintas) y de interconexión, así como una vitrina especial dedicada a los videojuegos, una industria muy ligada al ámbito informático que comenzó a tener importancia a finales de los años 70.

Finalmente, la exposición permanente del museo incorpora un conjunto de paneles informativos diseñados con un enfoque pedagógico que tratan diversos temas alrededor del mundo informático: la propia historia de la informática, los lenguajes de programación, el papel de la mujer en el desarrollo de la informática, algunas curiosidades sobre términos técnicos y dispositivos informáticos, la evolución de los dispositivos de almacenamiento que han ido incorporando los computadores, el concepto de mochila ecológica, los residuos electrónicos y su gestión, los materiales tóxicos presentes en los dispositivos electrónicos y el mundo de la publicidad en la informática. 


\section{Diseño de la experiencia}

La visita al Museo de Informática se planificó a principio de curso para así motivar al alumno, ya desde el inicio, en el estudio de la asignatura Estructura de Computadores y hacer que fuese observada desde un punto de vista distinto al experimentado en el aula y el laboratorio. Los conocimientos acerca de la evolución de los computadores a lo largo del tiempo, tanto en algunos aspectos y tan poco en otros, facilitan al alumno la percepción de los aspectos esenciales y básicos, de la importancia de las bases que se les transmiten en la asignatura y, principalmente, del motivo por el cual estos conocimientos son importantes. ¿Cuál es el común denominador de todo lo que se ha mostrado? ¿Cómo se harán las cosas en el futuro? El intento mismo de elaborar una respuesta a estas cuestiones debería permitir el establecimiento de las bases para una nueva percepción de la asignatura.

La visita al museo se organizó en dos partes o actividades claramente diferenciadas. La primera consistió en una breve conferencia que tuvo lugar en el salón de actos, en la que se mostró a los estudiantes no solo una perspectiva histórica de la informática - desde los primeros artefactos de cálculo hasta los computadores contemporáneos-, sino que además tuvo el objetivo de despertar su sensibilidad hacia aspectos sociológicos y medioambientales implicados. La charla se complementó con la proyección de vídeos donde se mostraban los principios del desarrollo de los computadores y su programación, anécdotas de la época, anuncios publicitarios y otros elementos visuales que hacían más amena la exposición. Esta primera parte tuvo una duración aproximada de una hora.

La segunda parte fue la visita propiamente dicha a los fondos que forman parte de la exposición permanente del museo y que, como se ha indicado, se encuentran expuestos en vitrinas y paneles informativos repartidos en las tres plantas de un mismo edificio. El alumno pudo efectuar el recorrido de la visita, deteniéndose para leer y asimilar los contenidos, en más o menos otra hora.

Teniendo en cuenta estos aspectos, en los próximos apartados describimos la manera en que organizamos la experiencia durante el pasado curso 2013/14 y el actual $2014 / 15$.

\subsection{Curso académico 2013/14}

En este curso la actividad tuvo carácter voluntario, de modo que el resultado de los cuestionarios no contribuyó en la nota final de la asignatura, aunque para incentivar la asistencia se les indicó que podría tenerse en cuenta en los casos extremos para redondear la nota al alza.

Para poder llevar a cabo tanto las experiencias de este tipo como los actos de evaluación, nuestro centro deja los lunes fuera de la planificación de horarios de los dos primeros cursos, por lo que en este caso se tiene asegurado el día adecuado en todos los grupos para llevar a cabo la actividad. Desde el punto de vista organizativo, el principal problema fue gestionar una visita para un elevado número de alumnos. Durante este curso la asignatura tuvo un total 
de 423 alumnos matriculados, organizados en 7 grupos de aula, todos ellos en horario de mañana excepto uno impartido por la tarde.

Para disponer de una previsión del número de alumnos interesados en participar en la actividad, previamente se pasaron unas listas en el aula para que se apuntaran y, de este modo, poder planificar el número de sesiones a realizar. En este curso, un total de 269 alumnos mostraron su interés por asistir al evento (el $64 \%$ de los matriculados). Dado que el aforo máximo del salón de actos es de 150 personas, se planificaron dos turnos. Para hacer un reparto sencillo se organizaron por grupos, cuatro en el primer turno y tres en el segundo, equilibrando así el número de asistentes. Las Figuras 1 y 2 recogen imágenes de esta parte.

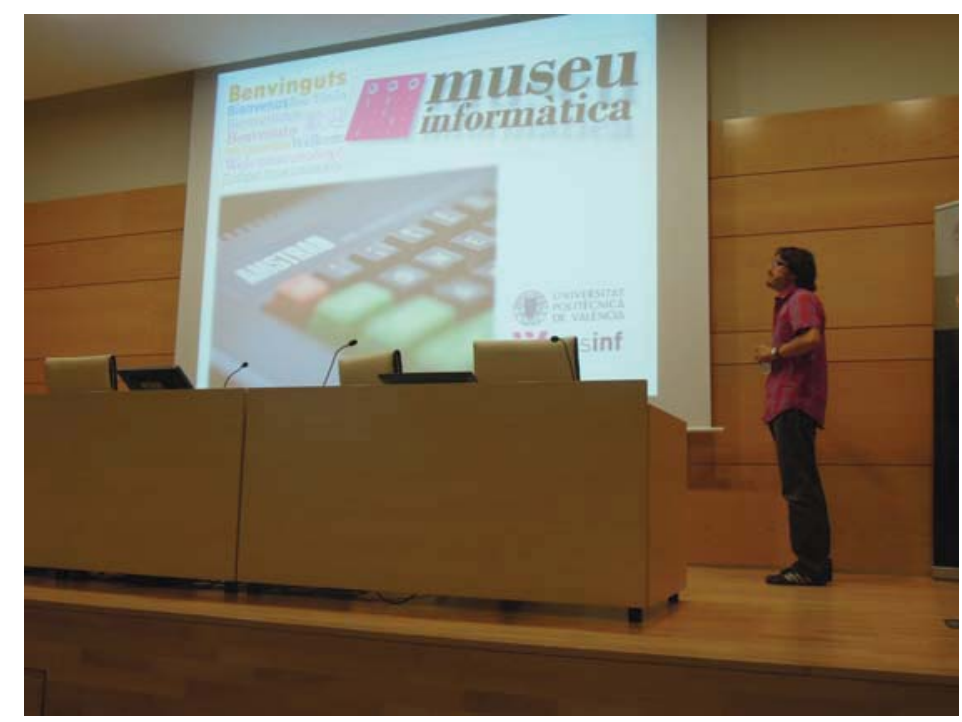

Figura 1. Presentación e introducción de la actividad.

Siguiendo la filosofía general de la metodología aplicada en esta asignatura, no hay actividades de obligado cumplimiento, sino que se deja al libre albedrío del estudiante la realización o no de las mismas, eso sí, siendo conscientes de que todas son importantes para su aprendizaje e incluso se tienen en cuenta para su evaluación. A pesar de ello, o quizá por ello, a priori se esperaba un elevado número de alumnos interesados en realizar la visita, por lo que, si bien no había más problema que el aforo del salón de actos para la primera parte, ya se preveía imposible la realización de visitas guiadas al uso, razón por la cual fue necesario convertir esta segunda parte en visitas autoguiadas.

Así pues, cada uno de los turnos establecidos estaba compuesto por más de cien alumnos. Como hemos indicado, aunque esto no representaba ningún problema para la parte prevista en el salón de actos, sí hacía inviable una visita 


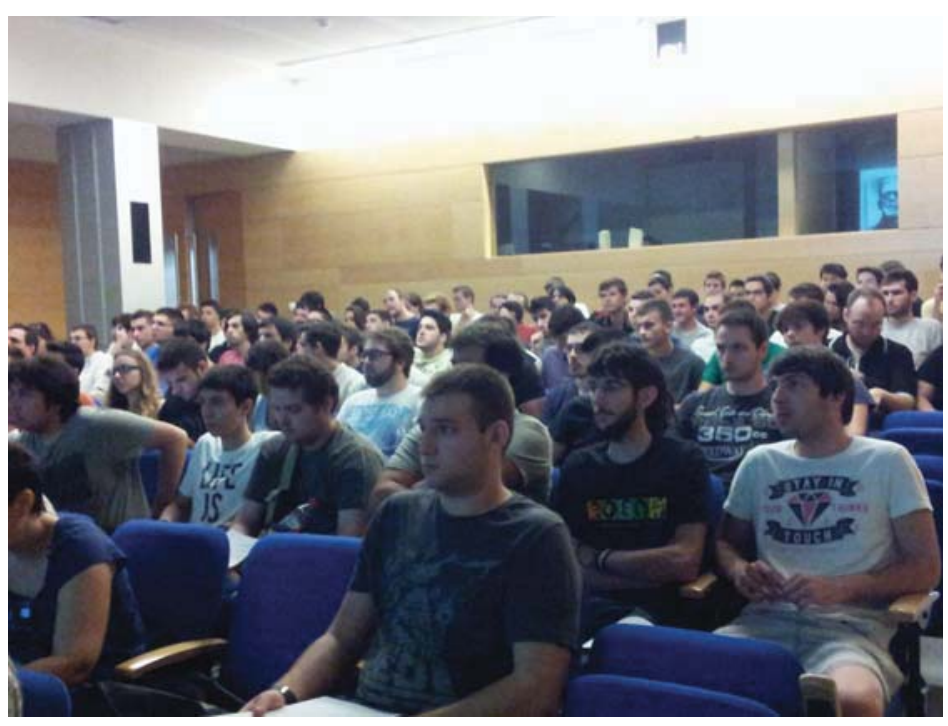

Figura 2. Alumnos asistentes a la actividad.

típica de grupo con guía. Como solución se propuso la visita al museo, de forma individual o por parejas, guiándola mediante un cuestionario que los alumnos iban resolviendo a su ritmo. Esto era totalmente viable porque todas las vitrinas del museo están documentadas mediante cartelas y, además, los paneles informativos complementarios son íntegramente autocontenidos. Los cuestionarios confeccionados trataban aquellos aspectos más interesantes y/o anecdóticos de los fondos de la exposición del museo. Con estos cuestionarios se pretendía mantener el interés en la exposición y, al mismo tiempo, guiar al alumno para que realizase una visita lo más completa posible. El cuestionario se entregó a la entrada de los alumnos al salón de actos, los contestaron durante la visita y, finalmente, los entregaron al profesor al término de la misma para su posterior evaluación. Las Figuras 3 y 4 ilustran sendos momentos de esta visita autoguiada en las que se puede apreciar cómo los alumnos interactúan con las vitrinas y los panales didácticos del museo.

El diseño de los cuestionarios requirió de una cuidada elaboración por nuestra parte, ya que además de cumplir con los objetivos principales de la actividad, pretendíamos evitar que solo un pequeño grupo de alumnos realizase el trabajo y luego comunicase las respuestas al resto. Por ello se diseñaron cuatro modelos de cuestionarios distintos. Previamente se elaboró un muestrario con un total de 100 preguntas relativas a los vídeos de la exposición y a las vitrinas de cada uno de los pisos del museo, así como de todos los paneles informativos: panel de lenguajes, paneles sobre el papel de la mujer en la informática, cuestiones de gestión de residuos y otros aspectos medioambientales y curiosidades. Con todas 


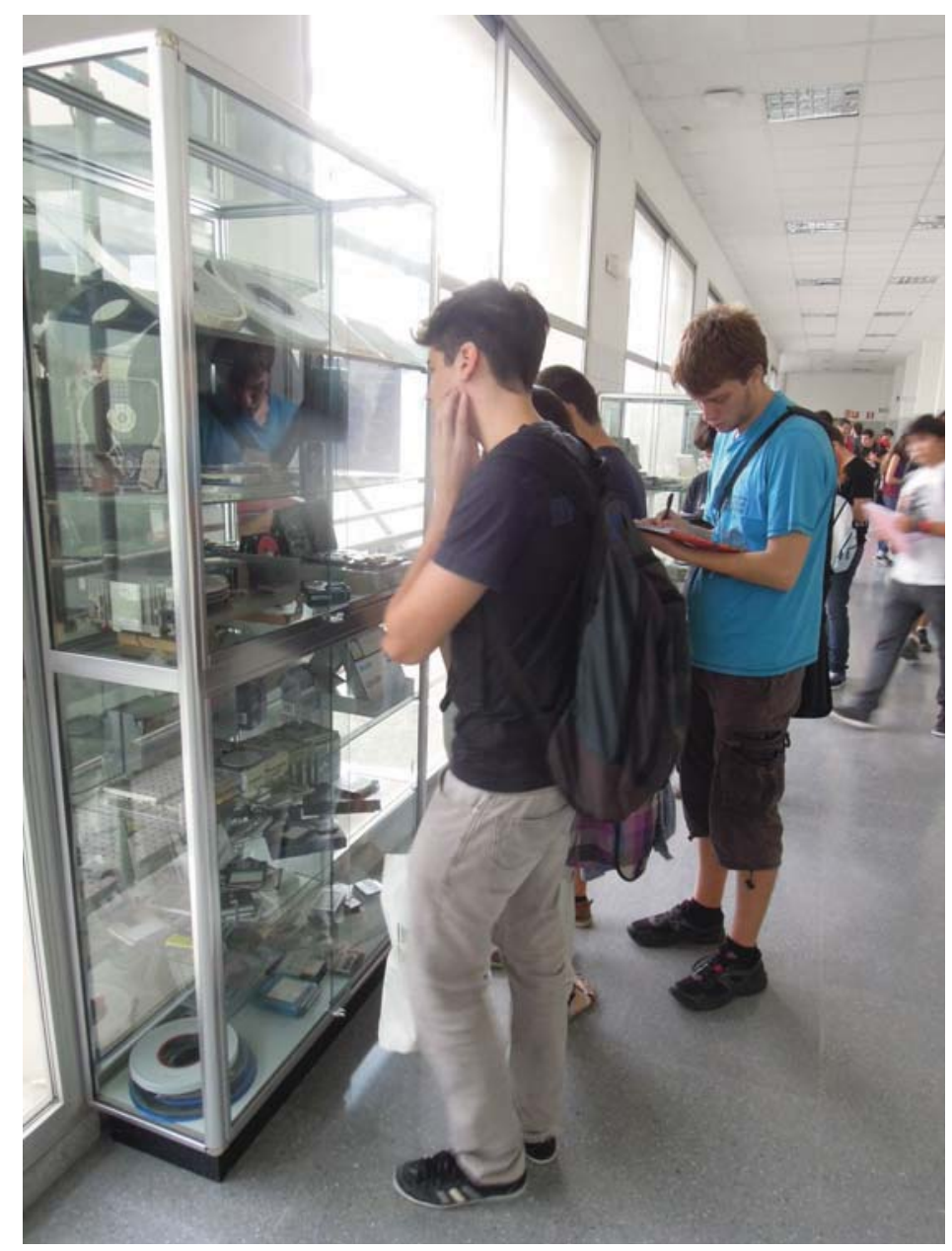

Figura 3. Visita: detalle de una de las vitrinas del museo. 


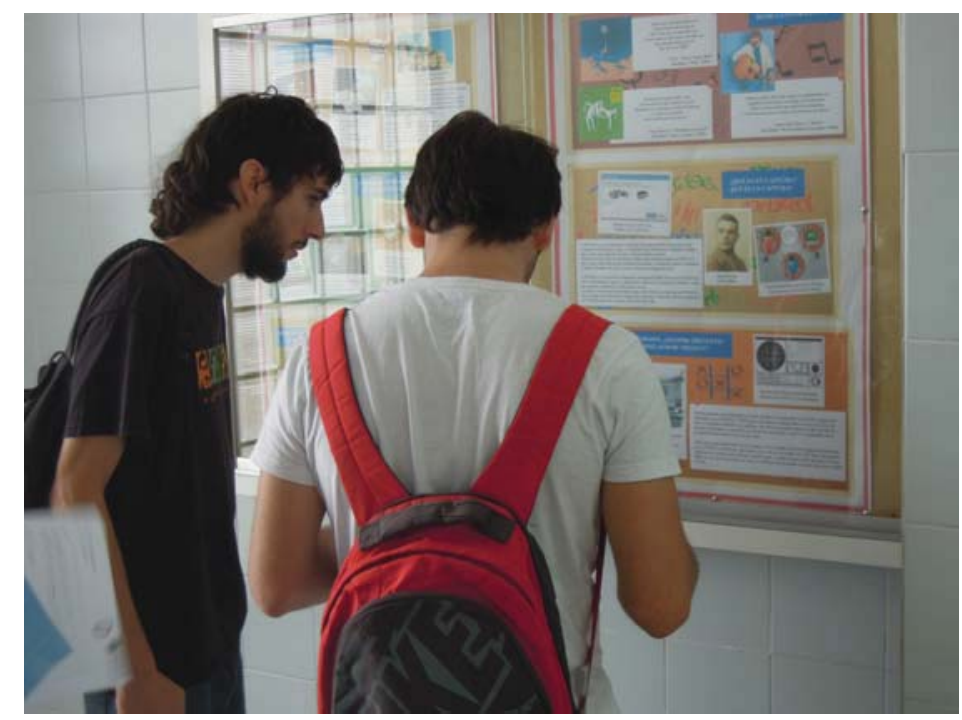

Figura 4. Visita: detalle de los paneles informativos.

las cuestiones se confeccionaron finalmente cuatro cuestionarios diferentes, con un total de 22 preguntas de respuesta corta cada uno de ellos. Casi todas ellas se podían responder buscando la información en las cartelas y paneles, por tratarse de nombres, fechas, etc. También, en algunos casos, era preciso realizar pequeños cálculos, como por ejemplo, para comparar la capacidad de un disco duro actual con el de uno de los años 80.

A continuación se muestran las cuestiones que integran uno de los cuatro cuestionarios que se facilitaron a los alumnos para responder durante el recorrido a lo largo de la exposición permanente del museo:

- ¿Qué numeración empleaba la máquina calculadora que Blaise Pascal diseñó en el año 1642? ¿Qué operaciones aritméticas podía llevar a cabo?

- ¿Con qué tipo de tecnología diseñó Charles Babbage su Máquina Analítica? ¿Mediante qué mecanismo se introducían los programas en este artefacto?

- ¿Con qué tecnología construyó Konrad Zuse su máquina Z1 en 1938 ?

- ¿Quién diseño en 1945 los componentes básicos de cualquier ordenador programable?

- El miniordenador DEC PDP-11 tiene en su arquitectura un rasgo innovador respecto a los ordenadores de su época. ¿De qué innovación se trata?

- ¿Cuántos kg pesan dos unidades HP 9121D de almacenamiento basada en discos flexibles? ¿Qué tipo de formato físico tiene este dispositivo?

- ¿Cómo se llama el procesador de textos que formaba parte de los programas distribuidos con el ordenador Amstrad PCW-8256?

- ¿Quién diseñó el ordenador Apple II y en qué año lo hizo? 
- ¿Qué quiere decir el término direct en el acrónimo DASD (direct acces storage device) que utiliza IBM para referirse a los discos duros magnéticos?

- ¿Cuántos platos tiene el dispositivo IBM 10SR (DASD, direct acces storage device)? ¿De qué tamaño son?

- ¿Para qué se usó fundamentalmente el ordenador Tatung Einstein TC-01?

- ¿Qué interfaz tienen en común los sistemas de almacenamiento de las estaciones de trabajo HP Apollo Series 700 y SGI Indigo ${ }^{2}$ IMPACT?

- ¿Qué quiere decir que el IBM PC tuviera una arquitectura abierta?

- ¿En qué procesador se basó el estándar MSX?

- ¿Qué sustancias químicas se utilizan para crear la capa magnetizable de los dispositivos de almacenamiento? Indique al menos dos.

- La impresora IBM 5211, ¿por qué se denomina impresora de líneas? ¿En qué año fue introducida en el mercado?

- ¿Cuál fue el primer computador construido según el principio de programa almacenado totalmente operativo? ¿En qué año se construyó?

- ¿Por qué organización y para qué tipo de entornos fue desarrollado el lenguaje de programación ADA?

- ¿Cómo se llama la mujer que promovió la fundación Mozilla y software libre?

- ¿De qué son acrónimo las siglas SPAM? ¿Quiénes fueron los responsables de su introducción en el ámbito de la informática?

- ¿Cuántos kg de sustancias químicas hacen falta para fabricar un ordenador de tamaño medio?

- El modelo Portable Personal Computer fue el segundo transportable de la empresa IBM. ¿Qué conocido personaje del cine se usó para publicitarlo? ¿Cuánto pesaba?

A partir del desarrollo de esta experiencia resulta interesante, al llegar a determinados puntos del temario, relacionar los contenidos explicados con lo visto y aprendido en el museo. Por ejemplo, podemos plantear la evolución de los procesadores desde los simples modelos pedagógicos hasta las tendencias actuales con una visión histórica y contextualizada. Así mismo, las características de las arquitecturas CISC y RISC son mucho más comprensibles con la perspectiva de los años, el papel del lenguaje ensamblador en el desarrollo de las arquitecturas es mucho más cercano, la evolución de las necesidades de cálculo científico y su relación con las aplicaciones gráficas y de realidad virtual actuales permite justificar mejor el esfuerzo realizado en el diseño de algoritmos y circuitos aritméticos y, por supuesto, es una ayuda inestimable a la hora de presentar los dispositivos periféricos y de almacenamiento. También, las constantes referencias a la evolución histórica de la informática creemos que ayudan a una mejor valoración de esta ingeniería por parte de nuestros estudiantes y a comprender los esfuerzos humanos y tecnológicos que, a lo largo de su corta vida, se han realizado y se siguen haciendo para convertirla en un elemento indispensable hoy en día.

\subsection{Curso académico 2014/15}

En el curso actual la visita al museo también fue planteada como actividad voluntaria, pero en esta ocasión se decidió que la calificación obtenida en ella 
fuera reflejada en la nota final del curso. Obviamente, a fin de no entrar en conflicto con la naturaleza voluntaria de la actividad, se propuso incrementar la nota final en 0.2 puntos sobre $10 \mathrm{y}$, consecuentemente, saturando en 10 la nota final. De esta manera, si un alumno no hubiera realizado la actividad, podría obtener igualmente la máxima nota participando en el resto de actos de evaluación de la asignatura.

En el presente curso nos propusimos mejorar el esquema organizativo atendiendo a la opinión expresadada por los alumnos del curso anterior, de modo que también se hicieron dos grupos para la visita, pero en este caso uno de mañana y otro de tarde. Por las mismas limitaciones de aforo — capacidad máxima del salón de actos - ambos grupos fueron dimensionados con 160 alumnos. El registro del alumnado se realizó a través de las facilidades proporcionadas por la plataforma digital PoliformaT para gestionar las asignaturas de la universidad. La visita fue realizada de un modo similar al curso anterior: una exposición introductoria y la visualización de varios vídeos divulgativos, seguida de la visita autoguiada por el cuestionario a rellenar. Teniendo presente que la nota obtenida en el cuestionario afectaría a la nota final del curso, se realizó un test online, con varias preguntas relativas a los contenidos del museo. Este test se realizó dos días después de la visita. Finalmente la nota de la actividad se obtuvo tomando la del test como punto de partida y redondeándola con la del cuestionario entregado el día de la visita.

Para poder preparar adecuadamente el test online se puso a disposición de los alumnos, a través de la página web del museo, una aplicación que planteaba baterías de diez preguntas sobre los contenidos de la exposición permanente y similares a las del test final ${ }^{4}$. Obviamente, el alumnos podía repetir este test las veces que considerase necesarias hasta tener la confianza suficiente para enfrentarse a la prueba evaluable. Adicionalmente se pasó una encuesta de satisfacción de la actividad en su conjunto. Esta encuesta se realizó al día siguiente. Para obtener nota en la actividad se estableció como necesario realizarla. Los alumnos fueron convenientemente avisados de estos requisitos, por correo electrónico y en la documentación aportada en la guía.

Con el propósito de motivar mejor a los estudiantes, durante la presentación oral se hizo énfasis en la importante tarea que tienen los ingenieros informáticos, y científicos en general, de contribuir al desarrollo de los computadores, haciendo hincapié en los avances tecnológicos que han provocado grandes cambios sociales durante las tres últimas décadas. Además se intentó relacionar estos aspectos con los principales contenidos de la charla, para demostrarles la utilidad de los conceptos que estudian. También se resaltó el papel, en muchas ocasiones decididamente relevante, que la mujer ha tenido en el ámbito de la infomática con algunos ejemplos fácilmente identificables, como el caso de los lenguajes de programación. Finalmente, se dio relevancia a la importancia del uso responsable de la tecnología y la conveniencia de reciclar por razones de salud y sostenibilidad medioambiental.

\footnotetext{
$\overline{{ }^{4} \text { http://museo.inf .upv.es/telosabes }}$
} 


\section{Evaluación de la experiencia}

A continuación se analizan los resultados de la experiencia, haciendo énfasis en las principales diferencias respecto al curso anterior.

En el presente curso 2014/15, el número de estudiantes de la asignatura asciende a 418. Como se ha mencionado, la actividad contó con una oferta de un total de 320 plazas distribuidas en dos grupos de 160 plazas cada uno: uno de mañana y otro de tarde. Finalmente, solamente 260 estudiantes asistieron a la visita, lo que representa el $81 \%$ de las plazas ofertadas. En el grupo de mañana se completaron todas las plazas, mientras que en el de tarde se cubrieron únicamente 100, esto es, el $63 \%$ de las plazas. Ello pone de manifiesto que pocos estudiantes están realmente interesados en la oferta de grupos de tarde aunque, en cambio, suelen ser bastante activos a la hora de reclamarlos. En general, se observa que poco más del $62 \%$ del número total de estudiantes mostraron interés por la actividad, lo cual desanima bastante a los responsables de organizarla, dado el enorme trabajo que conlleva la organización y las dificultades que entraña. Sin embargo, todos los asistentes entregaron el cuestionario utilizado para la visita autoguiada al término de la misma.

Respecto al test online concebido para evaluar el grado de aprovechamiento de la actividad, solo 197 estudiantes (el $76 \%$ ) lo completó. Entre estos, 35 estudiantes consiguieron un excelente (notas entre 9 y 10 puntos), 125 consiguieron notas entre 7 y 8 puntos, 29 consiguieron notas entre 5 y 6 puntos, mientras que solo 8 obtuvieron menos de 5 puntos. En el cuestionario escrito se obtuvieron resultados similares a los anteriores, pero con un menor número de excelentes, lo cual se justifica como consecuencia de un menor nivel de concentración, ello a pesar de que ninguna nota fue inferior a 5 , lo cual podría deberse al hecho de que el cuestionario se realizó en parejas.

Con el propósito de conocer la opinión de los estudiantes y compararla con los resultados obtenidos el pasado año, de modo que no permitiese identificar las fortalezas y debilidades de la experiencia, se llevó a cabo una encuesta de satisfacción de los estudiantes de forma anónima y a través de la plataforma educativa PoliformaT. La encuesta de realizó a lo largo de la misma semana de la visita al museo, de forma que los estudiantes pudieran albergar un recuerdo cercano de la experiencia. Lograr que los estudiantes respondan a cualquier tipo de encuesta es siempre complicado, especialmente si es online. Al objeto de asegurar un número suficiente de respuestas tal que nos permitiese extraer conclusiones válidas, la cumplimentación de la encuesta se impuso como condición para validar la participación de cada estudiante en la experiencia. A pesar de ello, únicamente 121 estudiantes, de los 260 que asistieron a la visita (sobre un $45 \%$ ), completaron la encuesta de satisfacción, lo cual significa que solo esos estudiantes podrán ver incrementada su nota final al cumplir todos los requisitos establecidos. Ello constituye un hecho bastante decepcionante, pues demuestra el escaso interés que los estudiantes prestan a cualquier actividad académica y lo escasamente motivados que están en general en sus estudios.

En el diseño de la encuesta se siguieron las recomendaciones acerca de encuestas de satisfacción presentadas en [2006]. En este sentido, tratamos de evaluar 
Cuadro 1. Cuestiones tratadas en la encuesta de evaluación.

Dimensión 1: Actividades previas a la visita

Q1 La charla previa a la visita al Museo de Informática me ha servido para descubrir aspectos sobre la historia de la informática que ignoraba

Q2 El documental sobre historia de la Informática me ha resultado atractivo y divulgativo

Q3 Los vídeos publicitarios me han servido para situar en su contexto las piezas del museo

Dimensión 2: Visita al museo

Q4 Las piezas y material que alberga el museo me han parecido interesantes

Q5 Algunos de los componentes, dispositivos y computadores que alberga el museo han despertado en mí la curiosidad

Q6 La visita me ha servido para comparar los usos de la informática actual con el pasado

Dimensión 3: Concienciación medioambiental y de género

Q7 Me parece bien que el museo se preocupe por los aspectos medioambientales relacionados con la informática

Q8 Creo que es necesario dar a conocer el papel que la mujer ha tenido y tiene en el desarrollo de la tecnología informática

Dimensión 4: Organización de la actividad

Q9 La forma en que se ha organizado la visita al museo me ha parecido adecuada

Q10El cuestionario que se ha cumplimentado al término de la visita me ha servido para apreciar detalles que, de otra forma, me habrían pasado desapercibidos

\begin{tabular}{l} 
Dimensión 5: Valoración global \\
\hline Q11 $\begin{array}{l}\text { Recomendaría a otras personas esta expe- } \\
\text { riencia o actividad educativa }\end{array}$ \\
\hline Q12 $\begin{array}{l}\text { Considero que la visita al Museo de Infor- } \\
\text { mática me ha servido de motivación para } \\
\text { el estudio de la asignatura de Estructura } \\
\text { de Computadores }\end{array}$ \\
\hline
\end{tabular}


cinco aspectos clave o dimensiones relativas a la experiencia, a saber, las actividades realizadas en el salón de actos previas a la visita (conferencia, documental y anuncios publicitarios), la visita al museo propiamente dicha, la organización de la experiencia, y la valoración global de la misma. Adicionalmente, este curso se ha creído interesante apreciar el grado de concienciación de los estudiantes respecto a los temas medioambiental y de género [2012], los cuales constituyen aspectos que el museo trata de promover. Con esta finalidad, se diseñó una encuesta con 12 preguntas y 5 opciones de respuesta: totalmente de acuerdo (TDA), más bien de acuerdo (MBA), indiferente (IND), más bien en desacuerdo (MBD), totalmente en desacuerdo (TED). También se incluye la opción de no sabe/no contesta (S/C). El Cuadro 1 muestra las diferentes cuestiones de la encuesta agrupadas por cada una de las dimensiones analizadas. En su diseño se trató de reflejar el objetivo perseguido por cada una de las dimensiones a través de la formulación de cuestiones muy concretas, de modo que al estudiante se le transmitiese de forma meridianamente clara la idea que subyace tras cada uno de los mencionados objetivos.

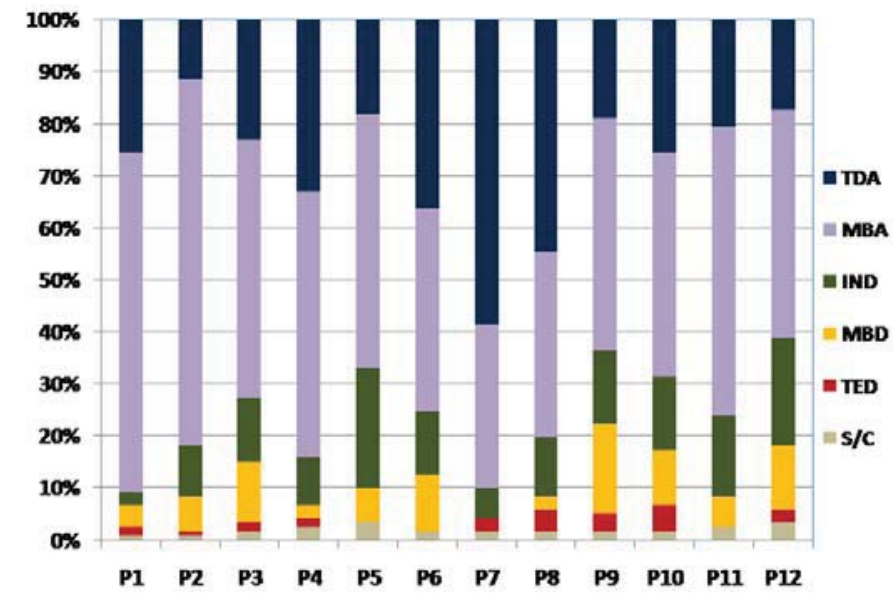

Figura 5. Resultado de la encuesta de evaluación.

Los resultados de la encuesta se muestran en la Figura 5. Del análisis de las respuestas se observa un incremento significativo en el porcentaje de estudiantes que reconoce que la visita le ha llevado a estudiar la materia de Estructura de Computadores con más interés (un $62 \%$ en comparación al $50 \%$ del curso anterior). Valoramos este resultado como muy positivo porque muestra el éxito de los esfuerzos llevados a cabo este curso para mejorar la motivación de nuestros estudiantes, lo que constituye el principal objetivo de la experiencia. El hecho de que esta actividad, por su propia naturaleza, no haya servido para motivar 
a una parte de estudiantes, no significa que éstos no puedan llegar a motivarse mediante otras actividades también programadas a este efecto a lo largo del curso como, por ejemplo, la visita a un clúster de altas prestaciones. De ahí la importancia de contar con un repertorio variado de actividades capaces de cubrir el máximo de sensibilidades.

También queremos resaltar el hecho de que un mayor porcentaje de alumnos manifestó estar de acuerdo con la organización de la visita (un $65 \%$ respecto al $60 \%$ el curso anterior). Nuevamente, ello muestra el éxito de los cambios introducidos este año en relación con la organización de la visita, fundamentalmente enfocados a subsanar algunas deficiencias detectadas en la primera edición, como por ejemplo la ausencia de grupo de tarde, que causó cierto malestar en un porcentaje significativo de los estudiantes el curso pasado. Ello muestra una vez más las enormes dificultades de organizar cualquier clase de actividad, experiencia de aprendizaje o metodología novedosa de enseñanza cuando se dirige a centenares de alumnos. A menudo, cuando a los profesores de universidad se nos critica por la falta de innovación en nuestra actividad docente, nadie tiene en cuenta las enormes complicaciones que conlleva la organización y gestión de grandes grupos de estudiantes.

El resto de preguntas de la encuesta fueron valoradas, en general, en términos similares al curso pasado. Entre ellas, cabe subrayar el hecho de que más del $90 \%$ de los estudiantes encontraron interesante la conferencia previa a la visita, así como que más del $76 \%$ recomendaría la experiencia, lo cual consideramos como un dato muy positivo. Sin embargo, hay algunos resultados curiosos, tal como el decremento observado en el porcentaje de estudiantes que reconoce que los contenidos del museo había despertado su curiosidad (únicamente un $67 \%$ en comparación al $75 \%$ alcanzado el curso anterior). Este hecho puede explicarse como consecuencia de que algunos estudiantes repitieron la visita por segunda vez, de modo que los contenidos apenas les sorprendieron. En cualquier caso, hemos de admitir que despertar la curiosidad de los estudiantes no es tarea fácil. Por tanto, valoramos de forma muy positiva que los contenidos del museo, al menos, hayan sido atractivos para un $85 \%$ de los estudiantes. Finalmente, cabe subrayar el hecho de que cerca de un $70 \%$ de los estudiantes reconoció la utilidad del cuestionario que tuvieron que cumplimentar al término de la visita.

Respecto a las dos nuevas preguntas incluidas en este curso en la encuesta, relativas al medioambiente y al papel de la mujer en el ámbito informático, más del $90 \%$ de los estudiantes valoraron positivamente la tarea de concienciación medioambiental desarrollada por el museo, mientras que un $80 \%$ valoró positivamente que esta institución constribuyera a subrayar el papel de la mujer en el desarrollo de los computadores.

\section{La retroinformática como perspectiva de futuro}

Del desarrollo y resultados de las actividades anteriores resulta patente la dificultad del profesorado universitario para lograr la motivación de sus alumnos. En particular, dentro del ámbito informático, creemos que los museos pueden 
representar, todavía, una gran oportunidad para despertar la curiosidad de una juventud posiblemente saturada de estímulos que, además, les llegan a través de las denominadas nuevas tecnologías y que, como sabemos, ya llevan muchos años integradas en nuestra vida cotidiana.

Dentro de la materia de la estructura de computadores, parcela docente que nos atañe en este trabajo, pensamos que la retroinformática puede seguir ayudándonos en nuestro objetivo motivador. En concreto, los primeros ordenadores personales (microordenadores) que inundaron las sociedades occidentales a principios de la década de los 80 del siglo XX, son susceptibles de convertirse en herramientas docentes con un atractivo especial. Estas máquinas de reducido tamaño, basadas en microprocesadores de 8 bits, programables en el lenguaje de programación BASIC, con su aspecto vintage, monitores de baja resolución algunos de ellos monocromos - , teclados curiosos y periféricos de almacenamiento basados en cintas magnéticas y discos flexibles - con formatos y capacidades diversas - , ofrecen la oportunidad de una manipulación bastante alejada de los dispositivos actuales como móviles inteligentes y tabletas y, en consecuencia, original a ojos de nuestros jóvenes estudiantes.

Esta pretendida originalidad, buscada interesadamente en el pasado, puede ayudar a situar a los alumnos ante ordenadores en los que, por ejemplo, conceptos sobre representación de la información y aritmética tengan que utilizarse en el ámbito de la programación en BASIC, donde pueden combinarse órdenes sencillas y directas como PRINT, formatos de impresión de datos en distintas bases y funciones matemáticas como FIX o ROUND, por citar solo algunas de las más conocidas. La interacción totalmente directa e inmediata con la memoria principal, del orden de pocos KB en estas máquinas, mediante lecturas y escrituras en determinadas zonas de la misma, también ofrece un entorno ágil y sencillo en el que estudiar el funcionamiento básico de esta unidad del ordenador.

Así mismo, los videojuegos también podrían convertirse en unos buenos aliados en nuestro empeño por motivar y enseñar. Tipologías de videojuegos no faltarán, ya que recordemos que la mayoría de estos microordenadores se utilizaron con este fin. En este sentido, la parsimoniosa carga de programas desde unidades de casete, la edición de código en un entorno limitado o el manejo incipiente de gráficos y sonidos podría resultar útil para ilustrar cómo, con una arquitectura sencilla y recursos muy limitados, los informáticos de la época desarrollaron aplicaciones interesantes y competitivas. A modo de ejemplo, la manipulación de un juego para conseguir, por ejemplo, vidas infinitas, puede ser un buen acicate para insistir en conceptos propios de nuestra materia: hay que saber en qué dirección de memoria está la instrucción máquina que decrementa el contador de vidas disponibles y cómo sobreescribir esta posición (los famosos POKE que difundían las revistas) con una instrucción máquina que no haga nada (habría que buscar la equivalente de la instrucción nop del MIPS R2000 en el repertorio de instrucciones del microprocesador de que se trate).

En este recorrido que estamos diseñando para el futuro próximo, contamos con la participación del Museo de Informática a través de su denominado Museo en vivo, un ámbito didáctico en el que se da cabida a un variado conjunto de mi- 
croordenadores de los años 80 del siglo XX todavía operativos. El inconveniente principal con que nos enfrentamos, más allá del planteamiento de la actividad didáctica en sí, es el reducido número de ordenadores disponibles, que está en torno a la decena, por lo que resultará complicado plantearla para un número elevado de alumnos. Una alternativa lógica podría venir de la mano de los emuladores disponibles de este tipo de máquinas, pero ello supondría la pérdida del contacto físico con los ordenadores reales, un valor intangible ofrecer al alumno y al que, al menos de momento, no queremos renunciar. Otra opción, de contenido mucho más ambicioso, vendría de la mano de un rediseño de los contenidos de la asignatura alrededor de la retroinformática y aplicarla en un grupo piloto con un número reducido de alumnos.

\section{Conclusiones}

La retroinformática y la evolución de los computadores nos presenta una magnífica oportunidad para motivar a nuestros alumnos en el estudio de materias básica como es el caso de la estructura de computadores y hacerles más sencillos y cercanos los contenidos. Con este fin, durante estos dos últimos académicos hemos organizado una serie de visitas y actividades alrededor del Museo de Informática de la Universitat Politècnica de València.

Uno de los principales problemas con los que se enfrenta el profesorado universitario en nuestro país a la hora de realizar actividades de innovación es el elevado número de alumnos a los que deben ir dirigidas, al que debemos añadir la escasez de medios humanos y materiales para apoyarlas. En este caso, las actividades desarrolladas han sido planteadas con carácter voluntario para más de 400 alumnos, aunque finalmente fueron alrededor de 260 los que mostraron interés en sumarse a ellas.

La experiencia se ha desarrollado en base a tres actividades básicas. La primera consiste en una charla sobre la historia de la informática, la evolución de los computadores y el software asociado, así como los aspectos sociales y medioambientales directamente relacionados. Todo ello ilustrado con la proyección de documentales cortos y anuncios publicitarios de la época. Esta primera parte se desarrolla en el salón de actos del centro, por lo que su aforo ha marcado el límite de alumnos a los que se puede ofrecer la actividad. Esta restricción nos ha obligado a planificar dos grupos para poder llevar a cabo la experiencia.

La segunda actividad consiste en una visita a la exposición permanente del Museo de Informática. Al ser tan elevado el número de asistentes no se puede plantear una visita guiada al uso, por lo que se ha recurrido a un recorrido autoguiado mediante un cuestionario específicamente diseñado para ello. Este planteamiento requiere una actitud proactiva por parte de los estudiantes y, hemos de reconocerlo, esta actitud no está presente en un elevado porcentaje de ellos, los cuales se muestran bastante apáticos y desinteresados. Esto queda patente en el bajo interés y dedicación con el que contestaron, usando lápiz y papel, las preguntas del cuestionario en ambas ediciones. 
Esta apatía se ha puesto más de manifiesto este curso ya que la actividad ha sido más exigente puesto que podía afectar de forma positiva a la nota final de curso. Por ello, en la segunda edición se ha planteado una tercera parte abordable de forma autónoma, en la que debían resolver un test online y que ha servido para obtener la nota de partida de la actividad global. Menos de la mitad de los participantes llegaron a completar este test y bastantes menos aún enviaron la encuesta de satisfacción asociada, a pesar de ser el elemento validador de la nota.

Sin embargo, y desde un punto de vista general, la experiencia ha sido positivamente valorada por un importante porcentaje de alumnos y también por parte del profesorado que se ha implicado en ella. Además, algunos puntos débiles detectados en la primera edición han sido subsanados este curso. Creemos que nos hemos acercado bastante al objetivo principal de la experiencia y hemos conseguido una mayor motivación de nuestros estudiantes mediante la retroinformática y la historia de los computadores. Además, hemos contribuido a difundir la historia de esta ciencia al mismo tiempo que creemos haber sembrado la semilla de la reflexión crítica sobre las consecuencias sociales y medioambientales derivadas del uso masivo de ordenadores y otros dispositivos inteligentes.

Finalmente, para fomentar una participación más activa de nuestros estudiantes, en un futuro próximo, nos planteamos aprovechar otros recursos y actividades promovidas por el Museo de Informática, como los talleres de retroprogramación, manejo y manipulación de videojuegos, y la participación en ciclos temáticos de cine.

\section{Referencias}

[2006] Guía para la realización de estudios de análisis de la demanda y encuestas de satisfacción, Ministerio de Administraciones Públicas. http://www.aeval.es/es/difusion_y_comunicacion/publicaciones/Guias/ Guias_Marco_General_Mejora_Calidad/guia_analisis.html (2006)

[2009] Memoria para la solicitud de verificación del título de Grado en Ingeniería Informática de la UPV, http://gradoinf.webs.upv.es/pdf/Memoria_grado_II. pdf (2009)

[2011] David A. Patterson, John L. Hennessy. Estructura y diseño de computadores. La interfaz hardware/software, Reverté, segunda edición (2011)

[2012] J. Abbate. Recoding gender: women's changing participation in computing. MIT Press, Cambridge, MA (2012)

[2012] Guía docente de la asignatura Estructura de Computadores, ETS d'Enginyeria Informàtica, 2012. http://www.upv.es/pls/oalu/sic_gdoc.get_content?P_ASI= 11552\&P_IDIOMA=c\&P_VISTA=\&P_TIT=156\&P_CACA=2013 (2012)

[2014] Ana Pont Sanjuán, Antonio Robles Martínez, Xavier Molero Prieto, Milagros Martínez Díaz. The Museum of Computer History - Teaching Support for Computer Organization Subjects 8th IT STAR Workshop on History of Computing, Hungría (2014)

[2014] Xavier Molero. El proyecto de difusión patrimonial del Museo de Informática de la UPV: el inicio de una nueva andadura, Congreso Internacional de Museos Universitarios, Madrid (2014). Pendiente de publicación. 